\title{
On the solvability of third-order three point systems of differential equations with dependence on the first derivative*
}

\author{
Feliz Minhós ${ }^{(\dagger)}$ and Robert de Sousa ${ }^{(\ddagger)}$ \\ ${ }^{(\dagger)}$ Departamento de Matemática, Escola de Ciências e Tecnologia, \\ Centro de Investigação em Matemática e Aplicações (CIMA), \\ Instituto de Investigação e Formação Avançada, \\ Universidade de Évora. Rua Romão Ramalho, 59, \\ 7000-671 Évora, Portugal \\ ${ }^{(\ddagger)}$ Faculdade de Ciências e Tecnologia, \\ Núcleo de Matemática e Aplicações (NUMAT), \\ Universidade de Cabo Verde. Campus de Palmarejo, \\ 279 Praia, Cabo Verde
}

\begin{abstract}
This paper presents sufficient conditions for the solvability of the third order three point boundary value problem

$$
\left\{\begin{array}{c}
-u^{\prime \prime \prime}(t)=f\left(t, v(t), v^{\prime}(t)\right) \\
-v^{\prime \prime \prime}(t)=h\left(t, u(t), u^{\prime}(t)\right) \\
u(0)=u^{\prime}(0)=0, u^{\prime}(1)=\alpha u^{\prime}(\eta) \\
v(0)=v^{\prime}(0)=0, v^{\prime}(1)=\alpha v^{\prime}(\eta) .
\end{array}\right.
$$

The arguments apply Green's function associated to the linear problem and the Guo-Krasnosel'skil theorem of compression-expansion cones. The dependence on the first derivatives is overcome by the construction of an adequate cone and suitable conditions of superlinearity/sublinearity near 0 and $+\infty$. Last section contains an example to illustrate the applicability of the theorem.
\end{abstract}

2010 Mathematics Subject Classification: 34B15, 34B18, 34B27, 34L30

${ }^{*}$ First author was supported by National Founds through FCT-Fundação para a Ciência e a Tecnologia, project SFRH/BSAB/114246/2016 
Keywords: Coupled systems, Green functions, Guo-Krasnosel'skiı̌ fixed-point in cones, positive solution.

\section{Introduction}

The solvability of systems of differential equations of second and higher order, with different types of boundary conditions has received an increasing interest in last years. See, for instance, [1, 3, 6, 7, 8, 9, 11, 12, 13, 16 and references therein. However systems where the nonlinearities can depend on the first derivatives are scarce (see [10]). Motivated by the works referred above, this paper contributes to fill that gap, applying cones theory to the third order three point boundary value problem

$$
\left\{\begin{array}{c}
-u^{\prime \prime \prime}(t)=f\left(t, v(t), v^{\prime}(t)\right) \\
-v^{\prime \prime \prime}(t)=h\left(t, u(t), u^{\prime}(t)\right) \\
u(0)=u^{\prime}(0)=0, u^{\prime}(1)=\alpha u^{\prime}(\eta) \\
v(0)=v^{\prime}(0)=0, v^{\prime}(1)=\alpha v^{\prime}(\eta) .
\end{array}\right.
$$

The non-negative continuous functions $f, h \in C\left([0,1] \times[0,+\infty)^{2},[0,+\infty)\right)$ verifying adequate superlinear and sublinear conditions, $0<\eta<1$ and the parameter $\alpha$ such that $1<\alpha<\frac{1}{\eta}$.

Third order differential equations can model various phenomena in physics, biology or physiology such as the flow of a thin film of viscous fluid over a solid surface (see[2, 18]), the solitary waves solution of the Korteweg-de Vries equation ([15]), or the thyroid-pituitary interaction (44).

A key point in our method is the fact that the Green's function associated to the linear problem and its first derivative are nonnegative and verify some adequate estimates. The existence of a positive and increasing solution of the system (1), is obtained by the well-known Guo-Krasnosel'skii theorem on cones compression-expansion. The dependence on the first derivatives is overcome by the construction of an adequate cone and suitable conditions of superlinearity/sublinearity near 0 and $+\infty$.

The paper is organized in the following way: In section 2 we present the integral equations equivalent to problem (1), the explicit form of the Green's function, and its derivative, and the definition of some functions used for its estimation. Section 3 contains the growth assumption on the nonlinearities and the main result to prove the existence of an increasing solution via cones theory. In last section an example illustrates the applicability of the theorem. 


\section{Preliminary results}

The pair of functions $(u(t), v(t)) \in\left(C^{3}[0,1],(0,+\infty)\right)^{2}$ is a solution of problem (1) if and only if $(u(t), v(t)) \in\left(C^{3}[0,1],(0,+\infty)\right)^{2}$ it is a solution of the following system of integral equations

$$
\left\{\begin{array}{l}
u(t)=\int_{0}^{1} G(t, s) f\left(s, v(s), v^{\prime}(s)\right) d s \\
v(t)=\int_{0}^{1} G(t, s) h\left(s, u(s), u^{\prime}(s)\right) d s
\end{array}\right.
$$

where $G(t, s)$ is the Green's function associated to problem (1), defined by

$$
G(t, s)=\frac{1}{2(1-\alpha \eta)}\left\{\begin{array}{cc}
\left(2 t s-s^{2}\right)(1-\alpha \eta)+t^{2} s(\alpha-1) & s \leq \min \{\eta, t\} \\
t^{2}(1-\alpha \eta)+t^{2} s(\alpha-1) & t \leq s \leq \eta \\
\left(2 t s-s^{2}\right)(1-\alpha \eta)+t^{2}(\alpha \eta-s) & \eta \leq s \leq t \\
t^{2}(1-s) & \max \{\eta, t\} \leq s
\end{array}\right.
$$

Next Lemmas provide some properties of the Green's functions and its derivative.

Lemma 1 (14])Let $0<\eta<1$ and $1<\alpha<\frac{1}{\eta}$. Then for any $(t, s) \in$ $[0,1] \times[0,1]$, we have $0 \leq G(t, s) \leq g_{0}(s)$, where

$$
g_{0}(s)=\frac{1+\alpha}{1-\alpha \eta} s(1-s) .
$$

Lemma 2 ([14])Let $0<\eta<1$ and $1<\alpha<\frac{1}{\eta}$. Then for any $(t, s) \in$ $\left[\frac{\eta}{\alpha}, \eta\right] \times[0,1]$, the Green function $G(t, s)$ verifies $G(t, s) \geq k_{0} g_{0}(s)$, where

$$
0<k_{0}:=\frac{\eta^{2}}{2 \alpha^{2}(1+\alpha)} \min \{\alpha-1,1\}<1 .
$$

The derivative of $G$ is given by

$$
\frac{\partial G}{\partial t}(t, s)=\frac{1}{(1-\alpha \eta)}\left\{\begin{array}{cc}
s(1-\alpha \eta)+t s(\alpha-1) & s \leq \min \{\eta, t\} \\
t(1-\alpha \eta)+t s(\alpha-1) & t \leq s \leq \eta \\
s(1-\alpha \eta)+t(\alpha \eta-s) & \eta \leq s \leq t \\
t(1-s) & \max \{\eta, t\} \leq s
\end{array}\right.
$$

and verifies the following lemmas: 
Lemma 3 For $0<\eta<1,1<\alpha<\frac{1}{\eta}$ and any $(t, s) \in[0,1] \times[0,1]$, we have $0 \leq \frac{\partial G}{\partial t}(t, s) \leq g_{1}(s)$, where

$$
g_{1}(s)=\frac{(1-s)}{(1-\alpha \eta)} .
$$

Proof. For $s \leq \min \{\eta, t\}$, we have

$$
\begin{aligned}
\frac{t(1-\alpha \eta)+t s(\alpha-1)}{(1-\alpha \eta)} & \leq \frac{s(1-\alpha \eta)+s(\alpha-1)}{(1-\alpha \eta)}=\frac{s(\alpha-\alpha \eta)}{(1-\alpha \eta)} \\
& =\frac{s \alpha(1-\eta)}{(1-\alpha \eta)} \leq \frac{s \alpha(1-s)}{(1-\alpha \eta)} \leq \frac{(1-s)}{(1-\alpha \eta)} .
\end{aligned}
$$

If $t \leq s \leq \eta$,

$\frac{t(1-\alpha \eta)+t s(\alpha-1)}{(1-\alpha \eta)}=\frac{t(1-\alpha \eta+s \alpha-s)}{(1-\alpha \eta)} \leq \frac{(1-\alpha \eta+\eta \alpha-s)}{(1-\alpha \eta)}=\frac{(1-s)}{(1-\alpha \eta)}$.

For $\eta \leq s \leq t$,

$$
\begin{aligned}
\frac{s(1-\alpha \eta)+t(\alpha \eta-s)}{(1-\alpha \eta)} & \leq \frac{s(1-\alpha \eta)+(\alpha \eta-s)}{(1-\alpha \eta)}=\frac{\alpha \eta(1-s)}{(1-\alpha \eta)} \\
& \leq \frac{\alpha s(1-s)}{(1-\alpha \eta)} \leq \frac{(1-s)}{(1-\alpha \eta)} .
\end{aligned}
$$

If $\max \{\eta, t\} \leq s$, then

$$
\frac{t(1-s)}{(1-\alpha \eta)} \leq \frac{s(1-s)}{(1-\alpha \eta)} \leq \frac{(1-s)}{(1-\alpha \eta)}
$$

So,

$$
\frac{\partial G}{\partial t}(t, s) \leq g_{1}(s):=\frac{(1-s)}{(1-\alpha \eta)}, \text { for }(t, s) \in[0,1] \times[0,1]
$$

Lemma 4 For $0<\eta<1,1<\alpha<\frac{1}{\eta}$ and any $(t, s) \in\left[\frac{\eta}{\alpha}, \eta\right] \times[0,1]$, the derivative of the Green function $\frac{\partial G}{\partial t}(t, s)$ verifies $\frac{\partial G}{\partial t}(t, s) \geq k_{1} g_{1}(s)$, with

$$
0<k_{1}:=\min \{\alpha \eta, \eta\}<1 \text {. }
$$

Proof. To find $k_{1}$ such that

$$
k_{1} g_{1}(s) \leq \frac{\partial G}{\partial t}(t, s)
$$

we evaluate it in each branch of $\frac{\partial G}{\partial t}(t, s)$ for $(t, s) \in\left[\frac{\eta}{\alpha}, \eta\right] \times[0,1]$, 
(i) For $s \leq \min \{\eta, t\}$, we must have

$$
k_{1} \frac{1-s}{1-\alpha \eta} \leq \frac{s(1-\alpha \eta)+t s(\alpha-1)}{1-\alpha \eta}
$$

that is

$$
k_{1} \leq \frac{s(1-\alpha \eta)+t s(\alpha-1)}{1-s} \leq \frac{\eta(1-\alpha \eta)+\eta(\alpha-1)}{1-\eta} \leq \frac{\eta(\alpha-\alpha \eta)}{1-\eta}=\alpha \eta<1 .
$$

(ii) If $t \leq s \leq \eta$, the inequality

$$
k_{1} \frac{1-s}{1-\alpha \eta} \leq \frac{t(1-\alpha \eta+s \alpha-s)}{1-\alpha \eta}
$$

holds for

$$
k_{1} \leq \frac{t(1-\alpha \eta+s \alpha-s)}{1-s} \leq \frac{\eta(1-\alpha \eta+\eta \alpha-\eta)}{1-\eta} \leq \frac{\eta(1-\eta)}{1-\eta}=\eta<1 .
$$

So, we can to define

$$
0<k_{1}=\min \{\alpha \eta, \eta\}<1
$$

The existence tool will be the well known Guo-Krasnoselskii results in expansive and compressive cones theory:

Lemma 5 ([5])Let $(E,\|\cdot\|)$ be a Banach space, and $P \subset E$ be a cone in $E$. Assume that $\Omega_{1}$ and $\Omega_{2}$ are open subsets of $E$ such that $0 \in \Omega_{1}, \overline{\Omega_{1}} \subset \Omega_{2}$.

If $T: P \cap\left(\overline{\Omega_{2}} \backslash \Omega_{1}\right) \rightarrow P$ is a completely continuous operator such that either

(i) $\|T u\| \leq\|u\|, u \in P \cap \partial \Omega_{1}$, and $\|T u\| \geq\|u\|, u \in P \cap \partial \Omega_{2}$,

or

(ii) $\|T u\| \geq\|u\|, u \in P \cap \partial \Omega_{1}$, and $\|T u\| \leq\|u\|, u \in P \cap \partial \Omega_{2}$, then $T$ has a fixed point in $P \cap\left(\overline{\Omega_{2}} \backslash \Omega_{1}\right)$. 


\section{Main result}

Consider the following growth assumptions

$$
\begin{gathered}
\limsup _{t \in[0,1],\|v\|_{C^{1}} \rightarrow 0} \frac{f\left(t, v, v^{\prime}\right)}{|v|+\left|v^{\prime}\right|}=0 \text { and } \limsup _{t \in[0,1],\|u\|_{C^{1} \rightarrow 0}} \frac{h\left(t, u, u^{\prime}\right)}{|u|+\left|u^{\prime}\right|}=0 ; \\
\liminf _{t \in[0,1],\|v\|_{C^{1}} \rightarrow+\infty} \frac{f\left(t, v, v^{\prime}\right)}{|v|+\left|v^{\prime}\right|}=+\infty \text { and } \liminf _{t \in[0,1],\|u\|_{C^{1}} \rightarrow+\infty} \frac{h\left(t, u, u^{\prime}\right)}{|u|+\left|u^{\prime}\right|}=+\infty ; \\
\liminf _{t \in[0,1],\|v\|_{C^{1}} \rightarrow 0} \frac{f\left(t, v, v^{\prime}\right)}{|v|+\left|v^{\prime}\right|}=+\infty \text { and } \liminf _{t \in[0,1],\|u\|_{C^{1}} \rightarrow 0} \frac{h\left(t, u, u^{\prime}\right)}{|u|+\left|u^{\prime}\right|}=+\infty ; \\
\limsup _{t \in[0,1],\|v\|_{C^{1}} \rightarrow+\infty} \frac{f\left(t, v, v^{\prime}\right)}{|v|+\left|v^{\prime}\right|}=0 \text { and } \limsup _{t \in[0,1],\|u\|_{C^{1}} \rightarrow+\infty} \frac{h\left(t, u, u^{\prime}\right)}{|u|+\left|u^{\prime}\right|}=0 .
\end{gathered}
$$

The main result is given by next theorem :

Theorem 6 Let $f, h:[0,1] \times[0,+\infty)^{2} \rightarrow[0,+\infty)$ be continuous functions such that assumptions $(A 1)$ and $(A 2)$, or $(A 3)$ and $(A 4)$, hold.

Then problem (1) has at least one positive solution $(u(t), v(t)) \in\left(C^{3}[0,1]\right)^{2}$, that is $u(t)>0, v(t)>0, \forall t \in[0,1]$.

Proof. Let $E=C^{1}[0,1]$ be the Banach space equipped with the norm $\|\cdot\|_{C^{1}}$, defined by $\|w\|_{C^{1}}:=\max \left\{\|w\|,\left\|w^{\prime}\right\|\right\}$ and $\|y\|:=\max _{t \in[0,1]}|y(t)|$.

Consider the set

$$
K=\left\{w \in E: w(t) \geq 0, \min _{t \in\left[\frac{\eta}{\alpha}, \eta\right]} w(t) \geq k_{0}\|w\|, \min _{t \in\left[\frac{\eta}{\alpha}, \eta\right]} w^{\prime}(t) \geq k_{1}\left\|w^{\prime}\right\|\right\},
$$

with $k_{0}$ and $k_{1}$ given by (4) and (5), respectively, and the operators $T_{1}$ : $K \rightarrow K$ and $T_{2}: K \rightarrow K$ such that

$$
\left\{\begin{array}{l}
T_{1} u(t)=\int_{0}^{1} G(t, s) f\left(s, v(s), v^{\prime}(s)\right) d s \\
T_{2} v(t)=\int_{0}^{1} G(t, s) h\left(s, u(s), u^{\prime}(s)\right) d s .
\end{array}\right.
$$

By (2), the solutions of the initial system (11) are fixed points of the operator $T:=\left(T_{1}, T_{2}\right)$.

First we show that $K$ is a cone. By definition of $K$ it is clear that $K$ is not identically zero or empty.

Consider $a, b \in \mathbb{R}^{+}$and $\forall x, y \in K$. Then

$$
x \in K \Rightarrow x \in E: x(t) \geq 0, \min _{t \in[0,1]} x(t) \geq k_{0}\|x\|, \min _{t \in[0,1]} x^{\prime}(t) \geq k_{1}\left\|x^{\prime}\right\|,
$$




$$
y \in K \Rightarrow y \in E: y(t) \geq 0, \min _{t \in[0,1]} y(t) \geq k\|y\|, \min _{t \in[0,1]} y^{\prime}(t) \geq k_{1}\left\|y^{\prime}\right\| .
$$

As $E$ is a vector space, consider the linear combination $a x+b y \in E$.

$$
\begin{aligned}
\min _{t \in[0,1]}(a x(t)+b y(t)) & =a \min _{t \in[0,1]} x(t)+b \min _{t \in[0,1]} y(t) \\
& \geq a k_{0}\|x\|+b k_{0}\|y\|=k_{0}(a\|x\|+b\|y\|) \\
& \geq k_{0}\|a x(t)+b y(t)\|,
\end{aligned}
$$

and

$$
\begin{aligned}
\min _{t \in[0,1]}(a x(t)+b y(t))^{\prime} & =a \min _{t \in[0,1]}(x(t))^{\prime}+b \min _{t \in[0,1]}(y(t))^{\prime} \\
& \geq a k_{1}\left\|x^{\prime}\right\|+b k_{1}\left\|y^{\prime}\right\|=k_{1}\left(a\left\|x^{\prime}\right\|+b\left\|y^{\prime}\right\|\right) \\
& \geq k_{0}\left\|(a x(t)+b y(t))^{\prime}\right\| .
\end{aligned}
$$

Therefore $a x+b y \in K$, that is $K$ is a cone.

Now we show that $T_{1}$ and $T_{2}$ are completely continuous, i.e, are equicontinuous and uniformly bounded.

For the reader's convenience the proof for $T_{1}$ will follow several steps and claims. The arguments for $T_{2}$ are analogous.

Step 1: $T_{1}$ and $T_{2}$ are well defined in $K$.

To prove that $T_{1} K \subset K$ consider $u \in K$.

As $G(t, s) \geq 0$ for $(t, s) \in[0,1] \times[0,1]$, It is clear that $T_{1} u(t) \geq 0$.

By Lemma 1, the positivity of $f$ and (6),

$$
0 \leq T_{1} u(t)=\int_{0}^{1} G(t, s) f\left(s, v(s), v^{\prime}(s)\right) d s \leq \int_{0}^{1} g_{0}(s) f\left(s, v(s), v^{\prime}(s)\right) d s .
$$

So,

$$
\left\|T_{1} u\right\| \leq \int_{0}^{1} g_{0}(s) f\left(s, v(s), v^{\prime}(s)\right) d s .
$$

From Lemma 2 and (7),

$$
\begin{aligned}
T_{1} u(t) & =\int_{0}^{1} G(t, s) f\left(s, v(s), v^{\prime}(s)\right) d s \\
& \geq k_{0} \int_{0}^{1} g_{0}(s) f\left(s, v(s), v^{\prime}(s)\right) d s \geq k_{0}\left\|T_{1} u\right\|, \text { for } t \in\left[\frac{\eta}{\alpha}, \eta\right],
\end{aligned}
$$

with $k_{0}$ given by (4). By Lemma 3 ,

$$
\left(T_{1} u(t)\right)^{\prime}=\int_{0}^{1} \frac{\partial G}{\partial t}(t, s) f\left(s, v(s), v^{\prime}(s)\right) d s \leq \int_{0}^{1} g_{1}(s) f\left(s, v(s), v^{\prime}(s)\right) d s,
$$


So,

$$
\left\|\left(T_{1} u\right)^{\prime}\right\| \leq \int_{0}^{1} g_{1}(s) f\left(s, v(s), v^{\prime}(s)\right) d s .
$$

By Lemma 4 and (8), it follows

$$
\begin{aligned}
\left(T_{1} u(t)\right)^{\prime} & =\int_{0}^{1} \frac{\partial G}{\partial t}(t, s) f\left(s, v(s), v^{\prime}(s)\right) d s \geq k_{1} \int_{0}^{1} g_{1}(s) f\left(s, v(s), v^{\prime}(s) d s\right. \\
& \geq k_{1}\left\|\left(T_{1} u\right)^{\prime}\right\|, \text { for } t \in\left[\frac{\eta}{\alpha}, \eta\right],
\end{aligned}
$$

and $k_{1}$ as in (5).

So $T_{1} K \subset K$. Analogously it can be shown that $T_{2} K \subset K$.

Assume that $(A 1)$ and $(A 2)$ hold.

By $(A 1)$, there exists $0<\delta_{1}<1$ such that, for $\left(t, v, v^{\prime}\right) \in[0,1] \times\left[0, \delta_{1}\right]^{2}$ and $\left(t, u, u^{\prime}\right) \in[0,1] \times\left[0, \delta_{1}\right]^{2}$,

$$
f\left(s, v(s), v^{\prime}(s)\right) \leq \varepsilon_{1}\left(|v(s)|+\left|v^{\prime}(s)\right|\right)
$$

and

$$
h\left(s, u(s), u^{\prime}(s)\right) \leq \varepsilon_{2}\left(|u(s)|+\left|u^{\prime}(s)\right|\right),
$$

with $\varepsilon_{1}$ and $\varepsilon_{2}$ to be defined forward.

Step 2: $\quad T_{1}$ and $T_{2}$ are completely continuous in $C^{1}[0,1]$.

$T_{1}$ is continuous in $C^{1}[0,1]$ as: $G(t, s), \frac{\partial G}{\partial t}(t, s)$ and $f$ are continuous.

Consider the set $B \subset K$, bounded in $C^{1}$, and let $u, v \in B$. Then there are $M_{1}, M_{2}>0$ such that $\|u\|_{C^{1}}<M_{1}$ and $\|v\|_{C^{1}}<M_{2}$.

Claim 2.1. $T_{1}$ is uniformly bounded in $C^{1}[0,1]$.

In fact, by (9), there are $M_{3}, M_{4}>0$ such that

$$
\begin{aligned}
\left\|T_{1} u\right\| & =\max _{t \in[0,1]}\left|T_{1} u(t)\right|=\max _{t \in[0,1]}\left|\int_{0}^{1} G(t, s) f\left(s, v(s), v^{\prime}(s)\right) d s\right| \\
& \leq \int_{0}^{1} \max _{t \in[0,1]}|G(t, s)|\left|f\left(s, v(s), v^{\prime}(s)\right)\right| d s \\
& \leq \int_{0}^{1} \max _{t \in[0,1]}|G(t, s)| \varepsilon_{1}\left(|v(s)|+\left|v^{\prime}(s)\right|\right) d s \\
& \leq 2 \varepsilon_{1}\|v\|_{C^{1}} \int_{0}^{1} \max _{t \in[0,1]}|G(t, s)|<M_{3}, \forall u \in B,
\end{aligned}
$$




$$
\begin{aligned}
\left\|\left(T_{1} u\right)^{\prime}\right\| & =\max _{t \in[0,1]}\left|\left(T_{1} u(t)\right)^{\prime}\right|=\max _{t \in[0,1]} \mid \int_{0}^{1} \frac{\partial G}{\partial t}(t, s) f\left(s, v(s), v^{\prime}(s) d s \mid\right. \\
& \leq \int_{0}^{1} \max _{t \in[0,1]}\left|\frac{\partial G}{\partial t}(t, s)\right| \mid f\left(s, v(s), v^{\prime}(s) \mid d s\right. \\
& \leq \int_{0}^{1} \max _{t \in[0,1]}\left|\frac{\partial G}{\partial t}(t, s)\right| \varepsilon_{1}\left(|v(s)|+\left|v^{\prime}(s)\right|\right) d s \\
& \leq 2 \varepsilon_{1}\|v\|_{C^{1}} \int_{0}^{1} \max _{t \in[0,1]}\left|\frac{\partial G}{\partial t}(t, s)\right| d s<M_{4}, \forall u \in B .
\end{aligned}
$$

Defining $M:=\max \left\{M_{3}, M_{4}\right\}$, then $\left\|T_{1} u\right\|_{C^{1}} \leq M$.

Claim 2.2. $T_{1}$ is equicontinuous in $C^{1}[0,1]$.

Let $t_{1}$ and $t_{2} \in[0,1]$. Without loss of generality suppose $t_{1} \leq t_{2}$. So

$$
\begin{aligned}
\left|T u\left(t_{1}\right)-T u\left(t_{2}\right)\right| & =\left|\int_{0}^{1}\left[G\left(t_{1}, s\right)-G\left(t_{2}, s\right)\right] f\left(s, v(s), v^{\prime}(s)\right) d s\right| \\
& \leq \int_{0}^{1}\left|G\left(t_{1}, s\right)-G\left(t_{2}, s\right)\right| \varepsilon_{1}\left(|v|+\left|v^{\prime}\right|\right) d s \\
& \leq 2 \varepsilon_{1}\|v\|_{C^{1}} \int_{0}^{1}\left|G\left(t_{1}, s\right)-G\left(t_{2}, s\right)\right| d s \rightarrow 0, \text { ast } t_{1} \rightarrow t_{2},
\end{aligned}
$$

and

$$
\begin{aligned}
\left|\left(T u\left(t_{1}\right)\right)^{\prime}-\left(T u\left(t_{2}\right)\right)^{\prime}\right| & =\left|\int_{0}^{1}\left[\frac{\partial G}{\partial t}\left(t_{1}, s\right)-\frac{\partial G}{\partial t}\left(t_{2}, s\right)\right] f\left(s, v(s), v^{\prime}(s)\right) d s\right| \\
& \leq \int_{0}^{1}\left|\frac{\partial G}{\partial t}\left(t_{1}, s\right)-\frac{\partial G}{\partial t}\left(t_{2}, s\right)\right| \varepsilon_{1}\left(|v|+\left|v^{\prime}\right|\right) d s \\
& \leq 2 \varepsilon_{1}\|v\|_{C^{1}} \int_{0}^{1}\left|\frac{\partial G}{\partial t}\left(t_{1}, s\right)-\frac{\partial G}{\partial t}\left(t_{2}, s\right)\right| d s \rightarrow 0, \text { as } t_{1} \rightarrow t_{2} .
\end{aligned}
$$

By the Arzèla-Ascoli's lemma $T_{1} B$ is relatively compact, that is, $T_{1}$ is compact .

Applying the same technique, using (10), it can be shown that $T_{2}$ is compact, too. Consequently $T$ is compact.

Next steps will prove that assumptions of Lemma 5 hold.

Step 3: $\left\|T_{1} u\right\|_{C^{1}} \leq\|u\|_{C^{1}}$, for some $\rho_{1}>0$ and $u \in K \cap \partial \Omega_{1}$ with $\Omega_{1}=\left\{u \in E:\|u\|_{C^{1}}<\rho_{1}\right\}$.

By $(A 1)$, define $0<\rho_{1}<1$ such that $\left(t, v, v^{\prime}\right) \in[0,1] \times\left[0, \rho_{1}\right]^{2}$ and $\left(t, u, u^{\prime}\right) \in[0,1] \times\left[0, \rho_{1}\right]^{2}$. 
From (9) and (10), choose $\varepsilon_{1}, \varepsilon_{2}>0$ sufficiently small such that

$$
\max \left\{\begin{array}{c}
\varepsilon_{1} \varepsilon_{2} \int_{0}^{1} g_{0}(s) d s \int_{0}^{1}\left(g_{0}(r)+g_{1}(r)\right) d r \\
\varepsilon_{1} \varepsilon_{2} \int_{0}^{1} g_{1}(s) d s \int_{0}^{1}\left(g_{0}(r)+g_{1}(r)\right) d r
\end{array}\right\}<\frac{1}{2} .
$$

If $u \in K$ and $\|u\|_{C^{1}}=\rho_{1}$, then, by Lemma 11, (2) and (11),

$$
\begin{aligned}
T_{1} u(t) & \leq \int_{0}^{1} g_{0}(s) \varepsilon_{1}\left(|v|+\left|v^{\prime}\right|\right) d s \\
& \leq \int_{0}^{1} g_{0}(s) \varepsilon_{1} \int_{0}^{1}\left(|G(t, r)|+\left|\frac{\partial G}{\partial t}(t, r)\right|\right)\left|h\left(r, u(r), u^{\prime}(r)\right)\right| d r d s \\
& \leq \int_{0}^{1} g_{0}(s) \varepsilon_{1} \int_{0}^{1}\left(g_{0}(r)+g_{1}(r)\right)\left|h\left(r, u(r), u^{\prime}(r)\right)\right| d r d s \\
& \leq \varepsilon_{1} \varepsilon_{2} \int_{0}^{1} g_{0}(s) d s \int_{0}^{1}\left(g_{0}(r)+g_{1}(r)\right)\left(|u(r)|+\left|u^{\prime}(r)\right|\right) d r \\
& \leq 2 \varepsilon_{1} \varepsilon_{2}\|u\|_{C^{1}} \int_{0}^{1} g_{0}(s) d s \int_{0}^{1}\left(g_{0}(r)+g_{1}(r)\right) d r<\|u\|_{C^{1}}
\end{aligned}
$$

and

$$
\begin{aligned}
\left(T_{1} u(t)\right)^{\prime} & =\int_{0}^{1} \frac{\partial G}{\partial t}(t, s) f\left(s, v(s), v^{\prime}(s) d s \leq \int_{0}^{1} g_{1}(s) \varepsilon_{1}\left(|v|+\left|v^{\prime}\right|\right) d s\right. \\
& \leq \int_{0}^{1} g_{1}(s) \varepsilon_{1} \int_{0}^{1}\left(g_{0}(r)+g_{1}(r)\right)\left|h\left(r, u(r), u^{\prime}(r)\right)\right| d r d s \\
& \leq 2 \varepsilon_{1} \varepsilon_{2}\|u\|_{C^{1}} \int_{0}^{1} g_{1}(s) d s \int_{0}^{1}\left(g_{0}(r)+g_{1}(r)\right) d r<\|u\|_{C^{1}},
\end{aligned}
$$

Therefore $\left\|T_{1} u\right\|_{C^{1}} \leq\|u\|_{C^{1}}$.

Step 4: $\left\|T_{1} u\right\|_{C^{1}} \geq\|u\|_{C^{1}}$, for some $\rho_{2}>0$ and $u \in K \cap \partial \Omega_{2}$ with $\Omega_{2}=\left\{u \in E:\|u\|_{C^{1}}<\rho_{2}\right\}$.

By $(A 2),\|v\|_{C^{1}} \rightarrow+\infty$ and $\|u\|_{C^{1}} \rightarrow+\infty$. Therefore there are several cases to be considered:

Case 4.1. Suppose that there exist $\theta_{1}, \theta_{2}>0$ such that $\|v\| \rightarrow+\infty$, $\left\|v^{\prime}\right\| \leq \theta_{1},\|u\| \rightarrow+\infty$ and $\left\|u^{\prime}\right\| \leq \theta_{2}$.

Consider $\rho>0$ such that for $\left(t, v, v^{\prime}\right) \in[0,1] \times[\rho,+\infty) \times\left[0, \theta_{1}\right]$ and $\left(t, u, u^{\prime}\right) \in[0,1] \times[\rho,+\infty) \times\left[0, \theta_{2}\right]$, we have

$$
f\left(t, v(t), v^{\prime}(t)\right) \geq \xi_{1}\left(|v(t)|+\left|v^{\prime}(t)\right|\right)
$$


and

$$
h\left(t, u(t), u^{\prime}(t)\right) \geq \xi_{2}\left(|u(t)|+\left|u^{\prime}(t)\right|\right),
$$

with $\xi_{1}, \xi_{2}$ such that

$$
\min \left\{\begin{array}{c}
\left(k_{0}\right)^{2} \xi_{1} \xi_{2} \int_{\frac{\eta}{\alpha}}^{\eta} g_{0}(s) d s \int_{\frac{\eta}{\alpha}}^{\eta}\left(k_{0} g_{0}(r)+k_{1} g_{1}(r)\right) d r \\
\xi_{1} \xi_{2} k_{0} k_{1} \int_{\frac{\eta}{q}}^{\eta} g_{0}(s) d s \int_{\frac{\eta}{\alpha}}^{\eta}\left(k_{0} g_{0}(r)+k_{1} g_{1}(r)\right) d r \\
\xi_{1} \xi_{2} k_{0} k_{1} \int_{\frac{\eta}{\alpha}}^{\eta} g_{1}(s) d s \int_{\frac{\eta}{\alpha}}^{\eta}\left(k_{0} g_{0}(r)+k_{1} g_{1}(r)\right) d r \\
\left(k_{1}\right)^{2} \xi_{1} \xi_{2} \int_{\frac{\eta}{\alpha}}^{\eta} g_{1}(s) d s \int_{\frac{\eta}{\alpha}}^{\eta}\left(k_{0} g_{0}(r)+k_{1} g_{1}(r)\right) d r \\
k_{0}\left(k_{0}+k_{1}\right) \xi_{1} \xi_{2} \int_{\frac{\eta}{\alpha}}^{\eta} g_{0}(s) d s \int_{\frac{\eta}{\alpha}}^{\eta}\left(k_{0} g_{0}(r)+k_{1} g_{1}(r)\right) d r \\
k_{0}\left(k_{0}+k_{1}\right) \xi_{1} \xi_{2} \int_{\frac{\eta}{\alpha}}^{\eta} g_{1}(s) d s \int_{\frac{\eta}{\alpha}}^{\eta}\left(k_{0} g_{0}(r)+k_{1} g_{1}(r)\right) d r
\end{array}\right\}>1
$$

with $k_{0}, k_{1}$ as in (4) and (5).

Let $u, v \in K$ such that $\|u\|_{C^{1}}=\rho_{2}$, where $\rho_{2}:=\max \left\{2 \rho_{1}, \frac{\rho}{k_{0}}, \frac{\rho}{k_{1}}\right\}$.

Then $\|u\|_{C^{1}}=\|u\|=\rho_{2}$ and $u(t) \geq k_{0}\|u\|_{C^{1}}=k_{0} \rho_{2} \geq \rho, t \in[0,1]$. Similarly, $\|v\|_{C^{1}}=\|v\|=\rho_{2}$ and $v(t) \geq k_{1}\|v\|_{C^{1}}=k_{0} \rho_{2} \geq \rho$.

By Lemma 2, (2) and (14),

$$
\begin{aligned}
T_{1} u(t) & \geq \int_{\frac{\eta}{\alpha}}^{\eta} G(t, s) f\left(s, v(s), v^{\prime}(s)\right) d s \\
& \geq k_{0} \int_{\frac{\eta}{\alpha}}^{\eta} g_{0}(s) f\left(s, v(s), v^{\prime}(s)\right) d s \geq k_{0} \xi_{1} \int_{\frac{\eta}{\alpha}}^{\eta} g_{0}(s) \quad\left(|v(s)|+\left|v^{\prime}(s)\right|\right) d s \\
& =k_{0} \xi_{1} \int_{\frac{\eta}{\alpha}}^{\eta} g_{0}(s) d s \int_{\frac{\eta}{\alpha}}^{\eta}\left(|G(t, r)|+\left|\frac{\partial G}{\partial t}(t, r)\right|\right)\left|h\left(r, u(r), u^{\prime}(r)\right)\right| d r \\
& \geq k_{0} \xi_{1} \xi_{2} \int_{\frac{\eta}{\alpha}}^{\eta} g_{0}(s) d s \int_{\frac{\eta}{\alpha}}^{\eta}\left(k_{0} g_{0}(r)+k_{1} g_{1}(r)\right) \quad\left(|u(r)|+\left|u^{\prime}(r)\right|\right) d r \\
& =k_{0} \xi_{1} \int_{\frac{\eta}{\alpha}}^{\eta} g_{0}(s) d s \int_{\frac{\eta}{\alpha}}^{\eta}\left(|G(t, r)|+\left|\frac{\partial G}{\partial t}(t, r)\right|\right)\left|h\left(r, u(r), u^{\prime}(r)\right)\right| d r \\
& \geq k_{0} \xi_{1} \xi_{2} \int_{\frac{\eta}{\alpha}}^{\eta} g_{0}(s) d s \int_{\frac{\eta}{\alpha}}^{\eta}\left(k_{0} g_{0}(r)+k_{1} g_{1}(r)\right) \quad\left(|u(r)|+\left|u^{\prime}(r)\right|\right) d r \\
\geq & k_{0} \xi_{1} \xi_{2} \int_{\frac{\eta}{\alpha}}^{\eta} g_{0}(s) d s \int_{\frac{\eta}{\alpha}}^{\eta}\left(k_{0} g_{0}(r)+k_{1} g_{1}(r)\right) \quad\left(\underset{r \in\left[\frac{\eta}{\alpha}, \eta\right]}{\min } u(r)+\min _{r \in\left[\frac{\eta}{\alpha}, \eta\right]} u^{\prime}(r)\right) d r \\
\geq & k_{0} \xi_{1} \xi_{2} \int_{\frac{\eta}{\alpha}}^{\eta} g_{0}(s) d s \int_{\frac{\eta}{\alpha}}^{\eta}\left(k_{0} g_{0}(r)+k_{1} g_{1}(r)\right) \quad\left(k_{0}\|u\|+k_{1}\left\|u^{\prime}\right\|\right) d r
\end{aligned}
$$




$$
\begin{aligned}
& \geq k_{0} \xi_{1} \xi_{2} \int_{\frac{\eta}{\alpha}}^{\eta} g_{0}(s) d s \int_{\frac{\eta}{\alpha}}^{\eta}\left(k_{0} g_{0}(r)+k_{1} g_{1}(r)\right) k_{0}\|u\|_{C^{1}} d r \\
& =k_{0}^{2}\|u\|_{C^{1}} \xi_{1} \xi_{2} \int_{\frac{\eta}{\alpha}}^{\eta} g_{0}(s) d s \int_{\frac{\eta}{\alpha}}^{\eta}\left(k_{0} g_{0}(r)+k_{1} g_{1}(r)\right) d r>\|u\|_{C^{1}},
\end{aligned}
$$

and, analogously,

$$
\begin{aligned}
\left(T_{1} u(t)\right)^{\prime} & \geq \int_{\frac{\eta}{\alpha}}^{\eta} \frac{\partial G}{\partial t}(t, s) f\left(s, v(s), v^{\prime}(s)\right) d s \\
& \geq k_{1} \int_{\frac{\eta}{\alpha}}^{\eta} g_{1}(s) f\left(s, v(s), v^{\prime}(s)\right) d s \geq k_{1} \xi_{1} \int_{\frac{\eta}{\alpha}}^{\eta} g_{1}(s)\left(|v(s)|+\left|v^{\prime}(s)\right|\right) d s \\
& \geq k_{1} \xi_{1} \xi_{2} \int_{\frac{\eta}{\alpha}}^{\eta} g_{1}(s) d s \int_{\frac{\eta}{\alpha}}^{\eta}\left(k_{0} g_{0}(r)+k_{1} g_{1}(r)\right) k_{0}\|u\|_{C^{1}} d r \\
& =k_{1} k_{0}\|u\|_{C^{1}} \xi_{1} \xi_{2} \int_{\frac{\eta}{\alpha}}^{\eta} g_{1}(s) d s \int_{\frac{\eta}{\alpha}}^{\eta}\left(k_{0} g_{0}(r)+k_{1} g_{1}(r)\right) d r>\|u\|_{C^{1}} .
\end{aligned}
$$

Therefore $\left\|T_{1} u\right\|_{C^{1}} \geq\|u\|_{C^{1}}$.

Case 4.2. Suppose that there exist $\theta_{3}, \theta_{4}>0$ such that $\left\|v^{\prime}\right\| \rightarrow+\infty$, $\|v\| \leq \theta_{3},\left\|u^{\prime}\right\| \rightarrow+\infty$ and $\|u\| \leq \theta_{4}$.

Consider $\rho>0$ such that for $\left(t, v, v^{\prime}\right) \in[0,1] \times\left[0, \theta_{3}\right] \times[\rho,+\infty)$ and $\left(t, u, u^{\prime}\right) \in[0,1] \times\left[0, \theta_{4}\right] \times[\rho,+\infty)$, conditions (12), (13) and (14) hold.

Let $u, v \in K$ such that $\|u\|_{C^{1}}=\rho_{2}$, where $\rho_{2}:=\max \left\{2 \rho_{1}, \frac{\rho}{k_{0}}, \frac{\rho}{k_{1}}\right\}$.

Then $\|u\|_{C^{1}}=\left\|u^{\prime}\right\|=\rho_{2}$ and $u^{\prime}(t) \geq k_{1}\left\|u^{\prime}\right\|=k_{1} \rho_{2} \geq \rho, t \in[0,1]$. Similarly, $\|v\|_{C^{1}}=\left\|v^{\prime}\right\|=\rho_{2}$ and $v^{\prime}(t) \geq k_{1}\left\|v^{\prime}\right\|=k_{1} \rho_{2} \geq \rho$.

As in the previous case, by Lemma 2, (2) and (14)

$$
\begin{aligned}
T_{1} u(t) & \geq \int_{\frac{\eta}{\alpha}}^{\eta} G(t, s) f\left(s, v(s), v^{\prime}(s)\right) d s \\
& \geq k_{0} \int_{\frac{\eta}{\alpha}}^{\eta} g_{0}(s) f\left(s, v(s), v^{\prime}(s)\right) d s \geq k_{0} \xi_{1} \int_{\frac{\eta}{\alpha}}^{\eta} g_{0}\left(|v(s)|+\left|v^{\prime}(s)\right|\right) d s \\
& =k_{0} \xi_{1} \int_{\frac{\eta}{\alpha}}^{\eta} g_{0}(s) d s \int_{\frac{\eta}{\alpha}}^{\eta}\left(|G(t, r)|+\left|\frac{\partial G}{\partial t}(t, r)\right|\right)\left|h\left(r, u(r), u^{\prime}(r)\right)\right| d r \\
& =k_{1} k_{0}\|u\|_{C^{1}} \xi_{1} \xi_{2} \int_{\frac{\eta}{\alpha}}^{\eta} g_{0}(s) d s \int_{\frac{\eta}{\alpha}}^{\eta}\left(k_{0} g_{0}(r)+k_{1} g_{1}(r)\right) d r>\|u\|_{C^{1}},
\end{aligned}
$$


and

$$
\begin{aligned}
\left(T_{1} u(t)\right)^{\prime} & \geq \int_{\frac{\eta}{\alpha}}^{\eta} \frac{\partial G}{\partial t}(t, s) f\left(s, v(s), v^{\prime}(s)\right) d s \\
& \geq k_{1} \int_{\frac{\eta}{\alpha}}^{\eta} g_{1}(s) f\left(s, v(s), v^{\prime}(s)\right) d s \geq k_{1} \xi_{1} \int_{\frac{\eta}{\alpha}}^{\eta} g_{1}(s) \quad\left(|v(s)|+\left|v^{\prime}(s)\right|\right) d s \\
& \geq k_{1} \xi_{1} \xi_{2} \int_{\frac{\eta}{\alpha}}^{\eta} g_{1}(s) d s \int_{\frac{\eta}{\alpha}}^{\eta}\left(k_{0} g_{0}(r)+k_{1} g_{1}(r)\right) k_{1}\|u\|_{C^{1}} d r \\
& =\left(k_{1}\right)^{2}\|u\|_{C^{1}} \xi_{1} \xi_{2} \int_{\frac{\eta}{\alpha}}^{\eta} g_{1}(s) d s \int_{\frac{\eta}{\alpha}}^{\eta}\left(k_{0} g_{0}(r)+k_{1} g_{1}(r)\right) d r>\|u\|_{C^{1}} .
\end{aligned}
$$

Case 4.3. Suppose that $\|v\| \rightarrow+\infty,\left\|v^{\prime}\right\| \rightarrow+\infty,\|u\| \rightarrow+\infty$ and $\left\|u^{\prime}\right\| \rightarrow+\infty$.

Consider $\rho>0$ such that for $\left(t, v, v^{\prime}\right) \in[0,1] \times[\rho,+\infty)^{2}$ and $\left(t, u, u^{\prime}\right) \in$ $[0,1] \times[\rho,+\infty)^{2}$, conditions (12), (13) and (14) hold.

Let $u, v \in K$ such that $\|u\|_{C^{1}}=\rho_{2}$, where $\rho_{2}:=\max \left\{2 \rho_{1}, \frac{\rho}{k_{0}}, \frac{\rho}{k_{1}}\right\}$.

Then $\|u\|_{C^{1}}=\|u\|=\left\|u^{\prime}\right\|=\rho_{2}$ and $u(t) \geq k_{0}\|u\|=k_{0} \rho_{2} \geq \rho, u^{\prime}(t) \geq$ $k_{1}\|u\|=k_{1} \rho_{2} \geq \rho, t \in[0,1]$. Similarly, $\|v\|_{C^{1}}=\|v\|=\left\|v^{\prime}\right\|=\rho_{2}, v(t) \geq$ $k_{0}\|v\|=k_{0} \rho_{2} \geq \rho$ and $v^{\prime}(t) \geq k_{1}\left\|v^{\prime}\right\|=k_{1} \rho_{2} \geq \rho$.

As before,

$$
\begin{aligned}
T_{1} u(t) & \geq k_{0} \xi_{1} \xi_{2} \int_{\frac{\eta}{\alpha}}^{\eta} g_{0}(s) d s \int_{\frac{\eta}{\alpha}}^{\eta}\left(k_{0} g_{0}(r)+k_{1} g_{1}(r)\right) \quad\left(|u(r)|+\left|u^{\prime}(r)\right|\right) d r \\
& \geq k_{0} \xi_{1} \xi_{2} \int_{\frac{\eta}{\alpha}}^{\eta} g_{0}(s) d s \int_{\frac{\eta}{\alpha}}^{\eta}\left(k_{0} g_{0}(r)+k_{1} g_{1}(r)\right) \quad\left(k_{0}+k_{1}\right)\|u\|_{C^{1}} d r \\
& =k_{0}\left(k_{0}+k_{1}\right)\|u\|_{C^{1}} \xi_{1} \xi_{2} \int_{\frac{\eta}{\alpha}}^{\eta} g_{0}(s) d s \int_{\frac{\eta}{\alpha}}^{\eta}\left(k_{0} g_{0}(r)+k_{1} g_{1}(r)\right) d r>\|u\|_{C^{1}},
\end{aligned}
$$

and

$$
\begin{aligned}
\left(T_{1} u(t)\right)^{\prime} & \geq k_{1} \int_{\frac{\eta}{\alpha}}^{\eta} g_{1}(s) f\left(s, v(s), v^{\prime}(s)\right) d s \geq k_{1} \xi_{1} \int_{\frac{\eta}{\alpha}}^{\eta} g_{1}(s)\left(|v(s)|+\left|v^{\prime}(s)\right|\right) d s \\
& =k_{1} \xi_{1} \int_{\frac{\eta}{\alpha}}^{\eta} g_{1}(s) d s \int_{\frac{\eta}{\alpha}}^{\eta}\left(|G(t, r)|+\left|\frac{\partial G}{\partial t}(t, r)\right|\right)\left|h\left(r, u(r), u^{\prime}(r)\right)\right| d r \\
& \geq k_{1} \xi_{1} \xi_{2} \int_{\frac{\eta}{\alpha}}^{\eta} g_{1}(s) d s \int_{\frac{\eta}{\alpha}}^{\eta}\left(k_{0} g_{0}(r)+k_{1} g_{1}(r)\right)\left(k_{0}+k_{1}\right)\|u\|_{C^{1}} d r \\
& =k_{1}\left(k_{0}+k_{1}\right)\|u\|_{C^{1}} \xi_{1} \xi_{2} \int_{\frac{\eta}{\alpha}}^{\eta} g_{1}(s) d s \int_{\frac{\eta}{\alpha}}^{\eta}\left(k_{0} g_{0}(r)+k_{1} g_{1}(r)\right) d r>\|u\|_{C^{1}} .
\end{aligned}
$$


The other cases follow the same arguments.

Therefore $\left\|T_{1} u\right\|_{C^{1}} \geq\|u\|_{C^{1}}$.

Then, by Lemma 5, $T_{1}$ has a fixed point in $K \cap\left(\overline{\Omega_{2}} \backslash \Omega_{1}\right)$.

By the same steps it can be proved that $T_{2}$ has a fixed point in $K \cap$ $\left(\overline{\Omega_{2}} \backslash \Omega_{1}\right)$, too.

Assume that $(A 3)$ and $(A 4)$ are verified.

Step 5: $\left\|T_{1} u\right\|_{C^{1}} \geq\|u\|_{C^{1}}$, for some $\rho_{3}>0$ and $u \in K \cap \partial \Omega_{3}$ with $\Omega_{3}=\left\{u \in E:\|u\|_{C^{1}}<\rho_{3}\right\}$.

By $(A 3)$, it can be chosen $\rho_{3}>0$ such that $\left(t, v, v^{\prime}\right) \in[0,1] \times\left[0, \rho_{3}\right]^{2}$, $\left(t, u, u^{\prime}\right) \in[0,1] \times\left[0, \rho_{3}\right]^{2}$, and there are $\xi_{3}, \xi_{4}>0$ with

$$
\begin{aligned}
f(t, v(t), v(t)) & \geq \xi_{3}\left(|v(t)|+\left|v^{\prime}(t)\right| \mid\right), \\
h\left(t, u(t), u^{\prime}(t)\right) & \geq \xi_{4}\left(|u(t)|+\left|u^{\prime}(t)\right| \mid\right)
\end{aligned}
$$

and

$$
\min \left\{\begin{array}{c}
\left(k_{0}\right)^{2} \xi_{3} \xi_{4} \int_{\frac{\eta}{\eta}}^{\eta} g_{0}(s) d s \int_{\frac{\eta}{\alpha}}^{\eta}\left(k_{0} g_{0}(r)+k_{1} g_{1}(r)\right) d r \\
k_{0} k_{1} \xi_{3} \xi_{4} \int_{\frac{\eta}{\alpha}}^{\frac{\eta}{\alpha}} g_{1}(s) d s \int_{\frac{\eta}{\alpha}}^{\eta}\left(k_{0} g_{0}(r)+k_{1} g_{1}(r)\right) d r \\
\xi_{3} \xi_{4} k_{0} k_{1} \int_{\frac{\eta}{\alpha}}^{\eta} g_{0}(s) d s \int_{\frac{\eta}{\alpha}}^{\eta}\left(k_{0} g_{0}(r)+k_{1} g_{1}(r)\right) d r \\
\left(k_{1}\right)^{2} \xi_{3} \xi_{4} \int_{\frac{\eta}{\alpha}}^{\eta} g_{1}(s) d s \int_{\frac{\eta}{\alpha}}^{\eta}\left(k_{0} g_{0}(r)+k_{1} g_{1}(r)\right) d r \\
\xi_{3} \xi_{4} k_{0}\left(k_{0}+k_{1}\right) \int_{\frac{\eta}{\eta}}^{\eta} g_{0}(s) d s \int_{\frac{\eta}{\eta}}^{\eta}\left(k_{0} g_{0}(r)+k_{1} g_{1}(r)\right) d r \\
k_{1}\left(k_{0}+k_{1}\right) \xi_{3} \xi_{4} \int_{\frac{\eta}{\alpha}}^{\frac{\eta}{\alpha}} g_{1}(s) d s \int_{\frac{\eta}{\alpha}}^{\eta}\left(k_{0} g_{0}(r)+k_{1} g_{1}(r)\right) d r
\end{array}\right\}>1 .
$$

Let $u \in K$ and $\|u\|_{C^{1}}=\rho_{3}$.

Case 5.1. Suppose $\|u\|_{C^{1}}=\|u\|=\rho_{3}$.

By Lemma 2, (2) and (15),

$$
\begin{aligned}
T_{1} u(t) & \geq \xi_{3} \int_{\frac{\eta}{\alpha}}^{\eta} G(t, s)\left(|v(s)|+\left|v^{\prime}(s)\right|\right) d s \\
& \geq \xi_{3} \xi_{4} k_{0} \int_{\frac{\eta}{\alpha}}^{\eta} g_{0}(s) d s \int_{\frac{\eta}{\alpha}}^{\eta}\left(k_{0} g_{0}(r)+k_{1} g_{1}(r)\right) k_{0}\|u\|_{C^{1}} d r \\
& =\xi_{3} \xi_{4}\left(k_{0}\right)^{2}\|u\|_{C^{1}} \int_{\frac{\eta}{\alpha}}^{\eta} g_{0}(s) d s \int_{\frac{\eta}{\alpha}}^{\eta}\left(k_{0} g_{0}(r)+k_{1} g_{1}(r)\right) d r>\|u\|_{C^{1}},
\end{aligned}
$$


and

$$
\begin{aligned}
\left(T_{1} u(t)\right)^{\prime} & \geq k_{1} \int_{\frac{\eta}{\alpha}}^{\eta} g_{1}(s) f\left(s, v(s), v^{\prime}(s)\right) d s \geq k_{1} \xi_{3} \int_{\frac{\eta}{\alpha}}^{\eta} g_{1}(s)\left(|v(s)|+\left|v^{\prime}(s)\right|\right) d s \\
& \geq k_{1} \xi_{3} \xi_{4} \int_{\frac{\eta}{\alpha}}^{\eta} g_{1}(s) d s \int_{\frac{\eta}{\alpha}}^{\eta}\left(k_{0} g_{0}(r)+k_{1} g_{1}(r)\right) k_{0}\|u\|_{C^{1}} d r \\
& =k_{0} k_{1}\|u\|_{C^{1}} \xi_{3} \xi_{4} \int_{\frac{\eta}{\alpha}}^{\eta} g_{1}(s) d s \int_{\frac{\eta}{\alpha}}^{\eta}\left(k_{0} g_{0}(r)+k_{1} g_{1}(r)\right) d r>\|u\|_{C^{1}} .
\end{aligned}
$$

Case 5.2. Suppose $\|u\|_{C^{1}}=\left\|u^{\prime}\right\|=\rho_{3}$.

By Lemma 2, (2) and (15)

$$
\begin{aligned}
T_{1} u(t) & \geq \xi_{3} \int_{\frac{\eta}{\alpha}}^{\eta} G(t, s)\left(|v(s)|+\left|v^{\prime}(s)\right|\right) d s \\
& \geq \xi_{3} k_{0} \int_{\frac{\eta}{\alpha}}^{\eta} g_{0}(s) \int_{\frac{\eta}{\alpha}}^{\eta}\left(|G(t, r)|+\left|\frac{\partial G}{\partial t}(t, r)\right|\right)\left|h\left(r, u(r), u^{\prime}(r)\right)\right| d r d s \\
& \geq \xi_{3} \xi_{4} k_{0} \int_{\frac{\eta}{\alpha}}^{\eta} g_{0}(s) d s \int_{\frac{\eta}{\alpha}}^{\eta}\left(k_{0} g_{0}(r)+k_{1} g_{1}(r)\right) k_{1}\|u\|_{C^{1}} d r \\
& =\xi_{3} \xi_{4} k_{0} k_{1}\|u\|_{C^{1}} \int_{\frac{\eta}{\alpha}}^{\eta} g_{0}(s) d s \int_{\frac{\eta}{\alpha}}^{\eta}\left(k_{0} g_{0}(r)+k_{1} g_{1}(r)\right) d r>\|u\|_{C^{1}},
\end{aligned}
$$

and

$$
\begin{aligned}
\left(T_{1} u(t)\right)^{\prime} & \geq k_{1} \xi_{3} \xi_{4} \int_{\frac{\eta}{\alpha}}^{\eta} g_{1}(s) d s \int_{\frac{\eta}{\alpha}}^{\eta}\left(k_{0} g_{0}(r)+k_{1} g_{1}(r)\right) \quad\left(|u(r)|+\left|u^{\prime}(r)\right|\right) d r \\
& \geq k_{1} \xi_{3} \xi_{4} \int_{\frac{\eta}{\alpha}}^{\eta} g_{1}(s) d s \int_{\frac{\eta}{\alpha}}^{\eta}\left(k_{0} g_{0}(r)+k_{1} g_{1}(r)\right)\left(\min _{r \in\left[\frac{\eta}{\alpha}, \eta\right]} u(r)+\min _{r \in\left[\frac{\eta}{\alpha}, \eta\right]} u^{\prime}(r)\right) d r \\
& =\left(k_{1}\right)^{2}\|u\|_{C^{1}} \xi_{3} \xi_{4} \int_{\frac{\eta}{\alpha}}^{\eta} g_{1}(s) d s \int_{\frac{\eta}{\alpha}}^{\eta}\left(k_{0} g_{0}(r)+k_{1} g_{1}(r)\right) d r>\|u\|_{C^{1}} .
\end{aligned}
$$

Case 5.3. Suppose $\|u\|_{C^{1}}=\|u\|=\left\|u^{\prime}\right\|=\rho_{3}$.

By Lemma 2, (2) and (15) 


$$
\begin{aligned}
T_{1} u(t) & \geq \int_{\frac{\eta}{\alpha}}^{\eta} G(t, s) f\left(s, v(s), v^{\prime}(s)\right) d s \\
& \geq \xi_{3} k_{0} \int_{\frac{\eta}{\alpha}}^{\eta} g_{0}(s) \int_{\frac{\eta}{\alpha}}^{\eta}\left(|G(t, r)|+\left|\frac{\partial G}{\partial t}(t, r)\right|\right)\left|h\left(r, u(r), u^{\prime}(r)\right)\right| d r d s \\
& \geq \xi_{3} \xi_{4} k_{0} \int_{\frac{\eta}{\alpha}}^{\eta} g_{0}(s) d s \int_{\frac{\eta}{\alpha}}^{\eta}\left(k_{0} g_{0}(r)+k_{1} g_{1}(r)\right) \quad\left(k_{0}\|u\|+k_{1}\left\|u^{\prime}\right\|\right) d r \\
& =\xi_{3} \xi_{4} k_{0}\left(k_{0}+k_{1}\right)\|u\|_{C^{1}} \int_{\frac{\eta}{\alpha}}^{\eta} g_{0}(s) d s \int_{\frac{\eta}{\alpha}}^{\eta}\left(k_{0} g_{0}(r)+k_{1} g_{1}(r)\right) d r>\|u\|_{C^{1}},
\end{aligned}
$$

and

$$
\begin{aligned}
\left(T_{1} u(t)\right)^{\prime} & \geq k_{1} \int_{\frac{\eta}{\alpha}}^{\eta} g_{1}(s) f\left(s, v(s), v^{\prime}(s)\right) d s \geq k_{1} \xi_{3} \int_{\frac{\eta}{\alpha}}^{\eta} g_{1}(s)\left(|v(s)|+\left|v^{\prime}(s)\right|\right) d s \\
& \geq k_{1} \xi_{3} \xi_{4} \int_{\frac{\eta}{\alpha}}^{\eta} g_{1}(s) d s \int_{\frac{\eta}{\alpha}}^{\eta}\left(k_{0} g_{0}(r)+k_{1} g_{1}(r)\right)\left(\min _{r \in\left[\frac{\eta}{\alpha}, \eta\right]} u(r)+\min _{r \in\left[\frac{\eta}{\alpha}, \eta\right]} u^{\prime}(r)\right) d r \\
& \geq k_{1}\left(k_{0}+k_{1}\right)\|u\|_{C^{1}} \xi_{3} \xi_{4} \int_{\frac{\eta}{\alpha}}^{\eta} g_{1}(s) d s \int_{\frac{\eta}{\alpha}}^{\eta}\left(k_{0} g_{0}(r)+k_{1} g_{1}(r)\right) d r>\|u\|_{C^{1}} .
\end{aligned}
$$

In any case, $\left\|T_{1} u\right\|_{C^{1}} \geq\|u\|_{C^{1}}$.

Step 6: $\left\|T_{1} u\right\|_{C^{1}} \leq\|u\|_{C^{1}}$, for some $\rho_{4}>0$ and $u \in K \cap \partial \Omega_{4}$ with $\Omega_{4}=\left\{u \in E:\|u\|_{C^{1}}<\rho_{4}\right\}$.

Let $u \in K$ and $\|u\|_{C^{1}}=\rho_{4}$.

Case 6.1. Suppose that $f$ and $h$ are bounded.

Then there is $N>0$ such that $f\left(t, v(t), v^{\prime}(t)\right) \leq N, h\left(t, u(t), u^{\prime}(t)\right) \leq N$, $\forall u, v \in[0, \infty)$.

Choose

$$
\rho_{4}=\max \left\{2 \rho_{3}, N \int_{0}^{1} g_{0}(s) d s, N \int_{0}^{1} g_{1}(s) d s\right\} .
$$

Then

$$
T_{1} u(t)=\int_{0}^{1} G(t, s) f\left(s, v(s), v^{\prime}(s)\right) d s \leq N \int_{0}^{1} g_{0}(s) d s \leq \rho_{4} \text {, for } t \in[0,1],
$$

and

$$
\left(T_{1} u(t)\right)^{\prime}=\int_{0}^{1} \frac{\partial G}{\partial t}(t, s) f\left(s, v(s), v^{\prime}(s)\right) d s \leq N \int_{0}^{1} g_{1}(s) d s \leq \rho_{4} \text {, for } t \in[0,1] .
$$


Thus, $\left\|T_{1} u\right\|_{C^{1}} \leq\|u\|_{C^{1}}$. Similarly $\left\|T_{2} v\right\|_{C^{1}} \leq\|v\|_{C^{1}}$ for any $v \in K$ and $\|v\|_{C^{1}}=\rho_{4}$.

Case 6.2. Consider that $f$ is bounded and $h$ is unbounded.

So, there is $N>0$ such that $f\left(t, v(t), v^{\prime}(t)\right) \leq N, \forall\left(v, v^{\prime}\right) \in[0,+\infty)^{2}$..

By $(A 4)$, there exists $M>0$ such that $h\left(t, u(t), u^{\prime}(t)\right) \leq \mu\left(|u(t)|+\left|u^{\prime}(t)\right|\right)$, whenever $|u(t)|+\left|u^{\prime}(t)\right| \geq M$, with $\mu$ verifying

$$
\max \left\{\mu \int_{0}^{1} g_{0}(s) d s, \mu \int_{0}^{1} g_{1}(s) d s\right\}<\frac{1}{2} .
$$

Setting

$$
p(r):=\max \left\{h\left(t, u(t), u^{\prime}(t)\right): t \in[0,1], 0 \leq u \leq r, 0 \leq u^{\prime} \leq r\right\},
$$

we have

$$
\lim _{r \rightarrow \infty} p(r)=+\infty
$$

Define

$$
\rho_{4}=\max \left\{2 \rho_{3}, M, N \int_{0}^{1} g_{0}(s) d s, N \int_{0}^{1} g_{1}(s) d s\right\} .
$$

such that $p\left(\rho_{4}\right) \geq p(r), 0 \leq r \leq \rho_{4}$. Then

$$
T_{1} u(t)=\int_{0}^{1} G(t, s) f\left(s, v(s), v^{\prime}(s)\right) d s \leq N \int_{0}^{1} g_{0}(s) d s \leq \rho_{4},
$$

and

$\left(T_{1} u(t)\right)^{\prime}=\int_{0}^{1} \frac{\partial G}{\partial t}(t, s) f\left(s, v(s), v^{\prime}(s)\right) d s \leq N \int_{0}^{1} g_{1}(s) d s \leq \rho_{4}$, for $t \in[0,1]$.

So, $\left\|T_{1} u\right\|_{C^{1}} \leq\|u\|_{C^{1}}$ for $\|u\|_{C^{1}}=\rho_{4}$.

Moreover, if $v \in K$ such that $\|v\|_{C^{1}}=\rho_{4}$, we have $|u(t)|+\left|u^{\prime}(t)\right| \geq \rho_{4} \geq$ $M$,

$$
h\left(t, u(t), u^{\prime}(t)\right) \leq \mu\left(|u(t)|+\left|u^{\prime}(t)\right|\right) \leq 2 \mu \rho_{4}
$$

and $p\left(\rho_{4}\right) \leq 2 \mu \rho_{4}$. Therefore

$$
\begin{aligned}
T_{2} v(t) & =\int_{0}^{1} G(t, s) h\left(s, u(s), u^{\prime}(s)\right) d s \leq \int_{0}^{1} g_{0}(s) p\left(\rho_{4}\right) d s \\
& \leq p\left(\rho_{4}\right) \int_{0}^{1} g_{0}(s) d s \leq 2 \mu \rho_{4} \int_{0}^{1} g_{0}(s) d s \leq \rho_{4},
\end{aligned}
$$


and

$$
\begin{aligned}
\left(T_{2} v(t)\right)^{\prime} & =\int_{0}^{1} \frac{\partial G}{\partial t}(t, s) h\left(s, u(s), u^{\prime}(s)\right) d s \leq \int_{0}^{1} g_{1}(s) p\left(\rho_{4}\right) d s \\
& \leq p\left(\rho_{4}\right) \int_{0}^{1} g_{1}(s) d s \leq 2 \mu \rho_{4} \int_{0}^{1} g_{1}(s) d s \leq \rho_{4} .
\end{aligned}
$$

Consequently $\left\|T_{2} v\right\|_{C^{1}} \leq\|v\|_{C^{1}}$ for $\|v\|_{C^{1}}=\rho_{4}$.

Case 6.3. Suppose that $f$ is unbounded and $h$ is bounded.

Then, there is $N>0$ such that $h\left(t, u(t), u^{\prime}(t)\right) \leq N, \forall\left(u, u^{\prime}\right) \in[0,+\infty)^{2}$., and, by $(A 4)$, there exists $M>0$ such that $f\left(t, v(t), v^{\prime}(t)\right) \leq \mu\left(|v|+\left|v^{\prime}\right|\right)$, for $\left(|v|+\left|v^{\prime}\right|\right) \geq M$ with $\mu$ satisfying (16).

Choosing $\rho_{4}$ as in (17), the arguments follow like in the previous case.

Case 6.4. Consider that $f$ and $h$ are unbounded.

By $(A 4)$, there is $M>0$ such that $f\left(t, v(t), v^{\prime}(t)\right) \leq \mu\left(|v|+\left|v^{\prime}\right|\right)$, $h\left(t, u(t), u^{\prime}(t)\right) \leq \mu\left(|u|+\left|u^{\prime}\right| \mid\right)$ for $|v|+\left|v^{\prime}\right| \geq M$ and $|u|+\left|u^{\prime}\right| \geq M$ with $\mu$ as in (16).

Setting

$$
\begin{aligned}
& p(r):=\max \left\{h\left(t, u(t), u^{\prime}(t)\right): t \in[0,1], 0 \leq u \leq r, 0 \leq u^{\prime} \leq r\right\}, \\
& q(r):=\max \left\{f\left(t, v(t), v^{\prime}(t)\right): t \in[0,1], 0 \leq v \leq r, 0 \leq v^{\prime} \leq r\right\}
\end{aligned}
$$

we have

$$
\lim _{r \rightarrow \infty} p(r)=+\infty \text { and } \lim _{r \rightarrow \infty} q(r)=+\infty
$$

Choose

$$
\rho_{4}=\max \left\{2 \rho_{3}, M\right\}
$$

such that $p\left(\rho_{4}\right) \geq p(r)$ and $q\left(\rho_{4}\right) \geq q(r)$ for $0 \leq r \leq \rho_{4}$.

Let $u, v \in K$ and $\|u\|_{C^{1}}=\|v\|_{C^{1}}=\rho_{4}$.

Arguing as in (18) it can be easily shown that $\left\|T_{1} u\right\|_{C^{1}} \leq\|u\|_{C^{1}},\left\|T_{2} v\right\|_{C^{1}} \leq$ $\|v\|_{C^{1}}$.

By Lemma 5 the operators $T_{1}, T_{2}$ has a fixed point in $K \cap\left(\overline{\Omega_{4}} \backslash \Omega_{3}\right)$, therefore $T=\left(T_{1}, T_{2}\right)$ has a fixed point $(u, v)$ which is a positive solution of the initial problem.

Moreover these functions $u$ and $v$ are given by

$$
\left\{\begin{array}{l}
u(t)=\int_{0}^{1} G(t, s) f\left(s, v(s), v^{\prime}(s)\right) d s \\
v(t)=\int_{0}^{1} G(t, s) h\left(s, u(s), u^{\prime}(s)\right) d s .
\end{array}\right.
$$

and are both increasing functions. 


\section{Example}

Consider the following third order nonlinear system

$$
\left\{\begin{array}{c}
-u^{\prime \prime \prime}(t)=\left(t^{2}+1\right)\left(e^{-v(t)}+\sqrt{\left|v^{\prime}(t)\right|}\right) \\
-v^{\prime \prime \prime}(t)=(u(t)+1)^{2} \arctan \left(\left|u^{\prime}(t)\right|+1\right) \\
u(0)=u^{\prime}(0)=0, u^{\prime}(1)=\frac{3}{2} u^{\prime}\left(\frac{1}{2}\right) \\
v(0)=v^{\prime}(0)=0, v^{\prime}(1)=\frac{3}{2} v^{\prime}\left(\frac{1}{2}\right) .
\end{array}\right.
$$

In fact this problem is a particular case of system (11) with

$$
\begin{aligned}
f\left(t, v(t), v^{\prime}(t)\right) & :=\left(t^{2}+1\right)\left(e^{-v(t)}+\sqrt{\left|v^{\prime}(t)\right|}\right) \\
h\left(t, u(t), u^{\prime}(t)\right) & :=(u(t)+1)^{2} \arctan \left(\left|u^{\prime}(t)\right|+1\right), \\
\eta & =\frac{1}{2} \text { and } \alpha=\frac{3}{2} .
\end{aligned}
$$

It can be easily check that the above functions are non-negative and verify the assumptions $(A 3)$ and $(A 4)$.

$f$ and $h$ are non-negative functions, because they are product of non negative functions. Note that, $\forall t$ and $\forall(u(t), v(t)) \in\left(C^{3}[0,1],(0,+\infty)\right.$, $\left(t^{2}+1\right) \geq 1, e^{-v(t)}=\frac{1}{e^{v(t)}} \geq 0, \sqrt{\left|v^{\prime}(t)\right|} \geq 0,(u(t)+1)^{2} \geq 1$ and for definition of arc tangent function, we know $\arctan : \mathbb{R} \rightarrow]-\frac{\pi}{2}, \frac{\pi}{2}[$ such that $\arctan (x) \rightarrow \frac{\pi}{2}$, as $x \rightarrow+\infty$ and $\arctan (x) \rightarrow-\frac{\pi}{2}$, as $x \rightarrow-\infty$. So, $f \geq 0, h \geq 0$.

Finally, as

$$
\begin{aligned}
\liminf _{t \in[0,1],\|v\|_{C^{1} \rightarrow 0}} \frac{\left(t^{2}+1\right)\left(e^{-v(t)}+\sqrt{\left|v^{\prime}(t)\right|}\right)}{|v|+\left|v^{\prime}\right|} & =+\infty, \\
\liminf _{t \in[0,1],\|u\|_{C^{1}} \rightarrow 0} \frac{(u(t)+1)^{2} \arctan \left(\left|u^{\prime}(t)\right|+1\right)}{|u|+\left|u^{\prime}\right|} & =+\infty
\end{aligned}
$$

conditionn $(A 3)$ kolds and 


$$
\begin{aligned}
\limsup _{t \in[0,1],\|v\|_{C^{1}} \rightarrow+\infty} \frac{\left(t^{2}+1\right)\left(e^{-v(t)}+\sqrt{\left|v^{\prime}(t)\right|}\right)}{|v|+\left|v^{\prime}\right|} & =0, \\
\limsup _{t \in[0,1],\|u\|_{C^{1}} \rightarrow+\infty} \frac{(u(t)+1)^{2} \arctan \left(\left|u^{\prime}(t)\right|+1\right)}{|u|+\left|u^{\prime}\right|} & =0
\end{aligned}
$$

assumptiom $(A 4)$ is satisfied.

Therefore, by Theorem 6, problem (19) has at least a positive solution $(u(t), v(t)) \in\left(C^{3}[0,1]\right)^{2}$, that is $u(t)>0, v(t)>0, \forall t \in[0,1]$.

\section{References}

[1] N.A. Asif, R.A. Khan, Positive solutions to singular system with fourpoint coupled boundary conditions, J. Math. Anal. Appl. 386 (2012) 848-861.

[2] F. Bernis, L.A. Peletier, Two problems from draining flows involving third-order ordinary differential equations, SIAM J. Math. Anal. 27 (2) (1996) 515-527.

[3] Y. Cui, J. Sun, On existence of positive solutions of coupled integral boundary value problems for a nonlinear singular superlinear differential system, Electron. J. Qual. Theory Differ. Equ. 41 (2012) 1-13.

[4] L. Danziger, G. Elmergreen, The thyroid-pituitary homeostatic mechanism, Bull. Math. Biophys. 18 (1956) 1-13.

[5] D. Guo, V. Lakshmikantham, Nonlinear problems in abstract cones,. Academic Press, 1988.

[6] J. Henderson, R. Luca, Boundary Value Problems for Systems of Differential, Difference and Fractional Equations, Positive Solutions, Elsevier, 2015.

[7] J. Henderson, R. Luca, Positive solutions for systems of nonlinear second-order multipoint boundary value problems, Math. Meth. Appl. Sci. , 37 (2014) 2502-2516

[8] G. Infante, F. Minhós, P. Pietramala, Non-negative solutions of systems of ODEs with coupled boundary conditions, Commun Nonlinear Sci Numer Simulat 17 (2012) 4952-4960. 
[9] G. Infante, P. Pietramala, Nonnegative solutions for a system of impulsive BVPs with nonlinear nonlocal BCs, Nonlinear Analysis: Modelling and Control, Vol. 19, No. 3, (2014) 413-431.

[10] T. Jankowski Nonnegative solutions to nonlocal boundary value problems for systems of second-order differential equations dependent on the first-order derivatives, Nonlinear Analysis 87 (2013) 83-101.

[11] P. Kang, Z. Wei, Existence of positive solutions for systems of bending elastic beam equations, Electron. J. Differential Equations, 19 (2012).

[12] E.K. Lee, Y.H. Lee, Multiple positive solutions of a singular EmdenFowler type problem for second-order impulsive differential systems, Bound. Value Probl., 2011, Art. ID 212980, 22 pp., 2011.

[13] Y. Li, Y. Guo, G. Li, Existence of positive solutions for systems of nonlinear third-order differential equations, Commun Nonlinear Sci Numer Simulat 14 (2009) 3792-3797.

[14] G. Li-Jun, S. Jian-Ping, Z. Ya-Hong. Existence of positive solutions for nonlinear third-order three-point boundary value problems. Nonlinear Anal. 68 (2008) 3151-3158.

[15] X. Liu, H. Chen, Y. Lü, Explicit solutions of the generalized KdV equations with higher order nonlinearity, Appl. Math. Comput., 171 (2005) 315-319.

[16] L. Liu, P. Kanga, Y. Wub, B. Wiwatanapataphee, Positive solutions of singular boundary value problems for systems of nonlinear fourth order differential equations, Nonlinear Anal. 68 (2008) 485-498.

[17] I. Stakgold, M. Holst, Green's functions and Boundary Value Problems. John Wiley and Sons, 3rd ed., New Jersey, 2011.

[18] E.O. Tuck, L.W. Schwartz, A numerical and asymptotic study of some third-order ordinary differential equations relevant to draining and coating flows, SIAM Rev. 32 (3) (1990) 453-469. 